\title{
Symptomatic Infrarenal Abdominal Aortic Pseudoaneurysm: A Case Report
}

\author{
Andrew Pan ${ }^{\mathrm{a}, \mathrm{f}}$, David Kang ${ }^{\mathrm{a}}$, Anne M. Lopez ${ }^{\mathrm{b}}$, Jessica L. Buickoc, \\ Miguel A. Lopez-Viego ${ }^{\mathrm{d}, \mathrm{e}}$
}

\begin{abstract}
A pseudoaneurysm is an abnormal focal dilation of a blood vessel contained within a fibrous capsule that is not composed of all three layers of the vessel (intima, media and adventitia). The wall of a pseudoaneurysm is therefore made up of the normal structures surrounding the artery and any thrombus lining the cavity. Factors predisposing the formation of pseudoaneurysms include trauma to the vessel wall, disrupted surgical anastomosis, unidentified injuries to vessel walls during catheterizations and endovascular procedures, and complications related to anticoagulation and antiplatelet therapy in patients undergoing invasive arterial interventions. We present here an unusual case of a 55-year-old Caucasian male with an abdominal aortic pseudoaneurysm formed secondary to a ruptured ulcerated atherosclerotic plaque inferior to the renal vessels. He presented with a month's history of worsening back and testicular pain cumulating to a visit to the Emergency Department. He underwent urgent surgical repair of the pseudoaneurysm followed by an uneventful recovery. This report discusses the etiology, presentation, management and the possible complications of an abdominal aortic pseudoaneurysm.
\end{abstract}

Keywords: Infrarenal aneurysm; Pseudoaneurysm; False aneurysm; Abdominal aorta aneurysm

\section{Introduction}

An abdominal aortic aneurysm is an abnormal focal local-

\footnotetext{
Manuscript accepted for publication May 24, 2013

${ }^{a}$ Nova Southeastern University College of Osteopathic Medicine, Fort Lauderdale, FL, USA

${ }^{b}$ The University of Michigan, Ann Arbor, MI, USA

${ }^{c}$ University of Miami Miller School of Medicine, Atlantis, FL, USA

${ }^{\mathrm{d}}$ Department of General and Vascular Surgery, JFK Medical Center, Atlantis, FL, USA

${ }^{\mathrm{e}}$ Bethesda Memorial Hospital, Boynton Beach, FL, USA

${ }^{\mathrm{f}}$ Corresponding author: Andrew S. Pan, 15265 NW Perimeter Dr,

Beaverton, OR 97006, USA. Email: Andrew.s.pan@hotmail.com
}

doi: http://dx.doi.org/10.14740/jcs187w ized extraluminal arterialized dilation in direct communication with the lumen of the aorta. Abdominal aortic aneurysms, whether symptomatic or asymptomatic, are usually true aneurysms, where all three layers of the vessel wall are intact. Less frequently, patients can present with signs and symptoms of a true aneurysm only to reveal through further workup that a pseudoaneurysm is the entity being treated. The wall of a pseudoaneurysm is typically made up an extraarterial fibrous capsule instead of the arterial intima, media and adventitia seen in true aneurysms. Since this extraarterial fibrous tissue lacks the elastic tissue of the normal arterial media, the persistent communication of the normal arterial lumen and the pseudoaneurysm lumen results in a gradual, often rapid, expansion of the aneurysm [1]. Since the pseudoaneurysm develops outside of the normal arterial wall, embolic and occlusive events are rare compared to true aneurysms [1]. Pseudoaneurysms, however, can cause symptoms due to direct pressure on adjacent structures such as nerves, blood vessels and hollow viscera [1].

As opposed to true aneurysms where the predisposing risk factors include smoking, male sex, advanced age, atherosclerosis and Caucasian race [2-5], the predisposing risk factors for pseudoaneurym formation include trauma to the vessel wall, surgical anastomosis, anticoagulation and female sex $[1,6]$. Anastomotic pseudoaneurysms are caused by an arterial leak developing between the vascular graft (autogenous or prosthetic) and the native artery [7]. With time, the surrounding fibrous capsule and lining thrombus stretches and forms the pseudoaneurysm [8]. The incidence of iatrogenic pseudoaneurysms following percutaneous arterial punctures has been reported to be as high as 7\% [9]. In a recent study by Norwood et al, half of the pseudoaneuryms occurred after arterial catheterization and one-third of the pseudoaneurysms were related to a previous surgical anastomosis [10]. In this case report, we present an unusual case of a pseudoaneurysm formed by a ruptured atherosclerotic plaque in the abdominal aorta.

\section{Case Report}

A 55-year-old Caucasian male arrived at the emergency 

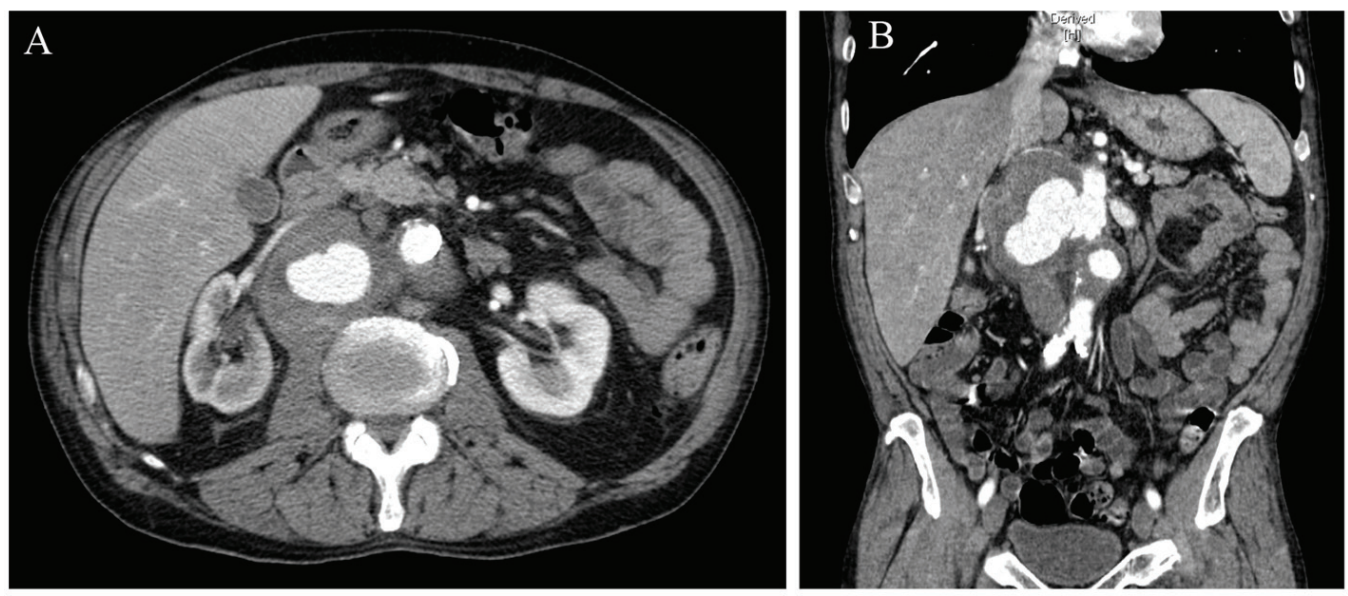

Figure 1. Pseudoaneurysm formed secondary to a ruptured atherosclerotic plaque lateralizing into the periaortic space. A: Cross-sectional view. B: Coronal view.

department complaining of severe right back and testicular pain. The patient reported that his pain had been ongoing for one month but had worsened for two days prior to visiting the emergency department. He reported the pain as stabbing, 10/10 on pain scale and radiating to his scrotum and abdomen. He denied dysuria, hematuria or any associated gastrointestinal symptoms. There was no report of lower extremity weakness or numbness, chest pain or shortness of breath. The past medical history included hypertension and dyslipidemia. No previous abdominal or pelvic operations were reported but the patient had undergone a previous cardiac catheterization through a femoral artery approach. The patient did have a history of tobacco and alcohol abuse.

His vital signs on admission included a blood pressure of $136 / 76 \mathrm{mmHg}$, pulse of $88 \mathrm{bpm}$, respiratory rate of $20 \mathrm{bpm}$, temperature of $36.7^{\circ} \mathrm{C}$ and a $\mathrm{SpO}_{2}$ of $100 \%$ on room air. His exam revealed a mild wheezing on lung auscultation bilaterally, a large left sided varicocele and small bilateral hydroceles. His abdomen was soft, non-distended and tender to palpation over a large pulsatile mid-abdominal mass. There was no evidence of jugular venous distention, cyanosis, leg edema or any neurologic or musculoskeletal abnormalities. The remainder of his physical exam was unremarkable.

The patient's metabolic panel values were within the reference ranges, with the exception of a sodium level of 132 $\mathrm{mmol} / \mathrm{dL}$ (136-145), BUN of $24 \mathrm{mg} / \mathrm{dL}$ (5-20), creatinine of $1.40 \mathrm{mg} / \mathrm{dL}(0.60-1.00)$ and magnesium of $1.4 \mathrm{mg} / \mathrm{dL}$ (1.82.4). His complete blood count, HbA1C, TSH and urinalysis were within normal limits.

A CT scan of the abdomen without contrast revealed a $9.2 \times 6.3 \times 8.7 \mathrm{~cm}$ mass with concentric high-density elements lateralizing into the periaortic space (Fig. 1). The density was located below the level of the kidney and had a mass effect on the right ureteral pelvic junction associated with a right hydronephrosis and anterior displacement of the right renal vein and right renal artery. Additional findings included a $1.7 \mathrm{~cm}$ complex cystic lesion in the upper pole of the right kidney, $1.5 \mathrm{~cm}$ low density focus in left hepatic lobe likely to be focal fat, mild splenomegaly, colonic diverticulosis and a few bibasilar lung nodules measuring $5 \mathrm{~mm}$ or less.

The patient underwent an urgent open repair of this symptomatic infrarenal abdominal aortic pseudoaneurysm. Intraoperatively, a defect was found on the right posterolateral aortic wall just inferior to the right renal artery where the rupture had occurred. Part of the pseudoneurysm wall was excised and sent to pathology. The infrarenal aorta was reconstructed using a tube $20 \mathrm{~mm}$ collagen impregnated Dacron graft sewn in place using running 3-0 Prolene sutures. The proximal anastamosis included the ostia of the right renal artery. After surgery, the patient was transferred to the ICU in stable condition. His recovery was uneventful.

\section{Discussion}

The presentation of pseudoaneuryms varies with the location and the vessel involved. Iatrogenic pseudoaneurysms often present shortly after arterial catherterization with pain and symptoms related to the tender, palpable, groin mass [1]. Pseudoaneuryms arising form major intra-abdominal or thoracic vessels are often asymptomatic until they become large enough to compress adjacent structures or rupture [1]. Our patient presented with a month's history of worsening back and testicular pain most likely due to the enlarging pseudoaneurysm. On admission, he presented with severe back and testicular pain, with a non-pulsatile abdomen that was tender to palpation. As illustrated in our case, the presence of pulsation of the mass is not a reliable indicator of a pseudoaneurysm, as up to one-quarter of false aneurysms are not pulsatile [1]. Since the pseudoaneurysm was slightly inferior to the kidneys, the pseudoaneurysm compressed the gonadal vein drainage leading to a prominent left-sided vari- 
cocele, hydroceles bilaterally and testicular pain. Our patient had risk factors associated with the development of a true abdominal aortic aneurysm, including male sex, smoking and Caucasian race. However, he did not have any of the predisposing factors in forming a pseudoaneurysm. He did not have a history of catheterizations, trauma to the vessel wall, nor a surgically created graft anastomosis in the area. In addition, he was of the male sex and was not on anticoagulation therapy. His pseudoaneurysm occurred secondary to a ruptured ulcerated atherosclerotic plaque. There had been reported cases of pseudoaneurysm resulting from spontaneous atherosclerotic plaque rupture, but most of them have been from the thoracic aorta instead of the abdominal aorta [11]. It is important to note that a pseudoaneurysm can become infected and a non-pulsatile infected pseudoaneurysm may present similarly to an abscess [1]. If the surgeon were to operate on the pseudoaneurysm under the impression that it was an abscess, the outcome could be disastrous. Therefore, one must maintain a clinical suspicion of a pseudoaneurysm.

\section{Conflict of Interest}

The above authors have no potential conflicts to disclose.

\section{References}

1. Parvin SD. Rare Vascular Disorders: A practical guide for the vascular specialist. Earnshaw JJ, consultant. Shrewsberry, UK; 2005. p 283-293.

2. Chaikof EL, Brewster DC, Dalman RL, Makaroun MS, Illig KA, Sicard GA, Timaran CH, et al. SVS practice guidelines for the care of patients with an abdominal aortic aneurysm: executive summary. J Vasc Surg.
2009;50(4):880-896.

3. Singh K, Bonaa KH, Jacobsen BK, Bjork L, Solberg S. Prevalence of and risk factors for abdominal aortic aneurysms in a population-based study: The Tromso Study. Am J Epidemiol. 2001;154(3):236-244.

4. Forsdahl SH, Singh K, Solberg S, Jacobsen BK. Risk factors for abdominal aortic aneurysms: a 7-year prospective study: the Tromso Study, 1994-2001. Circulation. 2009;119(16):2202-2208.

5. Kent KC, Zwolak RM, Egorova NN, Riles TS, Manganaro A, Moskowitz AJ, Gelijns AC, et al. Analysis of risk factors for abdominal aortic aneurysm in a cohort of more than 3 million individuals. J Vasc Surg. 2010;52(3):539-548.

6. Vuong PN, Berry C. The pathology of vessels. New York; 2002. p 220.

7. Chen FZ, Xu X, Fu WG, Wu ZG. Anastomotic false aneurysm following abdominal aortic aneurysmectomy and prosthetic grafting. Chin Med J (Engl). 1994;107(11):832-835.

8. Mii S, Mori A, Sakata H, Kawazoe N. Para-anastomotic aneurysms: incidence, risk factors, treatment and prognosis. J Cardiovasc Surg (Torino). 1998;39(3):259-266.

9. Morgan R, Belli AM. Current treatment methods for postcatheterization pseudoaneurysms. J Vasc Interv Radiol. 2003;14(6):697-710.

10. Norwood MG, Lloyd GM, Moore S, Patel N, Panditi S, Sayers RD. The changing face of femoral artery false aneurysms. Eur J Vasc Endovasc Surg. 2004;27(4):385388.

11. Vasquez J, Poultsides GA, Lorenzo AC, Foster JE, Drezner AD, Gallagher J. Endovascular stent-graft placement for nonaneurysmal infrarenal aortic rupture: a case report and review of the literature. J Vasc Surg. 2003;38(4):836-839. 